\title{
The Design and Development of a Full Range of Display System for 3D Images Based on Android Smartphone
}

\author{
Haomin Song ${ }^{1, a^{*}}$ and Duanqing $\mathrm{Xu}^{2, \mathrm{~b}}$ \\ ${ }^{1}$ The second middle school of Hang Zhou, Zhejiang, China \\ ${ }^{2}$ Zhejiang University, China \\ ahdlianyi@sina.com, bluochen333@gmail.com
}

Keywords: Android systems; 3D-images; Full range of display; Cloud computing

\begin{abstract}
With the popularity of smart phones, the demand, that people wish to get a full view of an interested object by smart phones, is increasing. Aimed at the demand, we design and develop such a system, which can all-round displays a 3D object for Android smart phones. This system could be very useful in variety fields, such as tourism, electric mall, educating etc.
\end{abstract}

\section{Introduction}

Nowadays, the main way for people to get information is through the graphic images. This way, however, with the progress of science and technology, does not meet the need of people any longer. Just like scenery or items in electric mall, people require more details, which mean full range of display of 3D images is the trend of development in the future.

Compared to the large side of the computer, smart phones are more convenient to carry. In other words, they are indispensable for people to get information at present. So if a full range of display system for 3D images could be designed, we can enjoy the 'real scenery' or observe 'real items' in electric mall through the phones in anywhere. Before developing this system, the rapid 3D modeling and full range of display, these two problems, must be solved.

On the other hand, this system, based on smart phones, would run lots of energy because of its complicated calculations, which is the fatal flaw for users. So we must use recourses outside of phones, such as cloud computing.

\section{Designs and Steps}

First of all, we use the cloud computing to realize the function of complicated images processing, and it is important to select the cloud. Then, some unnecessary parts in the model would be cut. After that, it will be saved in the server. Finally, 3D models would be showed in phones. Users could stretch or draw, and move the models in any direction, which provides users great convenient. Fig. 1 shows the steps below (next page).

\section{Building 3D Models Based on Cloud Computing}

The process of building 3D models is very complicated, that involves large amount of calculation, and runs lots of energy. But using cloud computing could solve this problem, and it is also a way to make the fast generation of 3D model as well. There are many 3D cloud processing websites, but we choose Autodesk 123D Catch (for free) of Autodesk company, which could transform the digital photos to realistic 3D models within the powerful cloud computing.

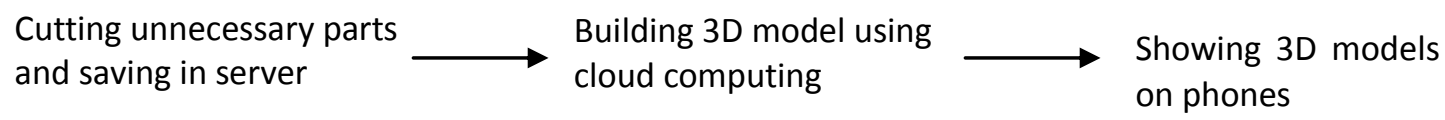

Figure. 1 Steps of the System Design 


\section{Theory and Process of Generation of 3D Models}

Here is the major theory of the photo-reconstruction in the cloud:

Find the characteristics of every photo and match between every two photos. This step is order to distinguish precisely the partly characteristics of items, and match them quickly. But it is difficult actually for many reasons. And the SIFT method referred to David Lowe ${ }^{[1]}$ is the most commonly used in practice.

According to the result of matching, the scene information will be calculated using projective theorem. This step is also called Structure from Motion ${ }^{[2]}$. The accuracy is the standard of the results. It is also called Sparse Reconstruction sometimes.

Put the scene information and the origin photos together to get the 3D points cloud of the items in the photos. Multi-view Stereo Reconstruction is available with the scene information. This progress also has a lot of calculation, because of the precision photos. So the execution efficiency is one of the standards to measure the algorism besides the accuracy and integrity of the construction. These points decide the quality of the point cloud. PMVS algorism ${ }^{[3]}$ is the best one for Multi-view Stereo Reconstruction. It is also called Dense Reconstruction.

Building the 3D models according to 3D point cloud. It is usually using Poisson Surface Reconstruction to line the point in cloud.

Building Models Using Autodesk 123D Catch. Taking photos. It is better to take photos in the same background and in different angles in order to get more details. 40 to 50 photos are enough for a good result, like Fig.2.

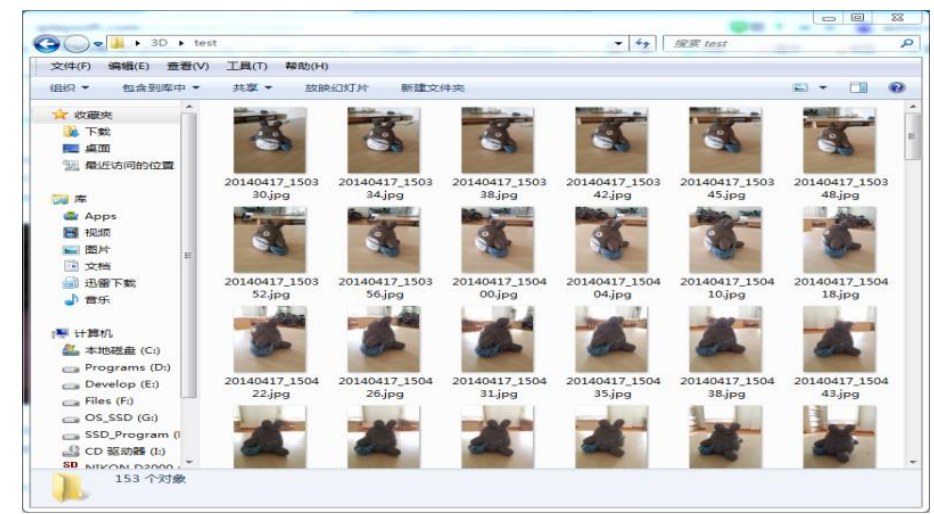

Figure. 2 Photos

Unloading photos. Using the software of 123D Catch, unload the photos to the internet.

Generation of model. 3D models would generate after the photos unloading, like Fig.3shows.

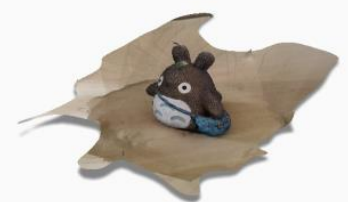

Figure. 3 The Unprocessed Model

Handling. Use Mesh lab to cut the unnecessary parts off, and save it in the server, like Fig.4 shows.

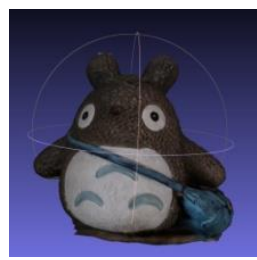

Figure. 4 The Processed Model 


\section{Development Based on Android System}

Android System and Its development environment. In October, 2007, Google and Open Handset Alliance released the platform of smart phones ${ }^{[4]}$.The development environment of Android system is in the table1.

Table 1 Development Environment of Android System

\begin{tabular}{ccc}
\hline Operating System & Java $<$ TM>version & Compiler \\
\hline Windows 7 Ultimate(64 bit) & $1.7 .0 \_25$ & Eclipse Java IDE
\end{tabular}

The Choice of 3D Engine and the Function Development. The commonly used 3D engines include: ORGE, Irrlicht, and min3D. Among them, min3D is based on OpenGL ES 1.x, which is designed for Android system. So we choose it as the basic engine. Considering its simple and single function, we make the secondary development to show 3D models better.

Min3D engine could only read object, material and texture files encapsulated inside APK. But the object (.obj) and material (.mtl) files must be stored in the res/raw directory without suffix meanwhile textures files must be stored in the res/drawable directory, which gives rise to great difficult to read the files loading from the server and limits the application.

Aimed at this problem, we add the method of analyzing the outside-model to the ObjParser.java, which allows the alternative path to the third party model in the programs, and reading the model files loading from the server.

We make a background for the model instead of the black, which makes the 3D model show livelier.

System Engineering Structure. Fig.5 shows the engineering structure directory diagram of the Android system. Packing these files makes Android software. The important parts are scr directory, res directory, and AndroidManifest.xml.

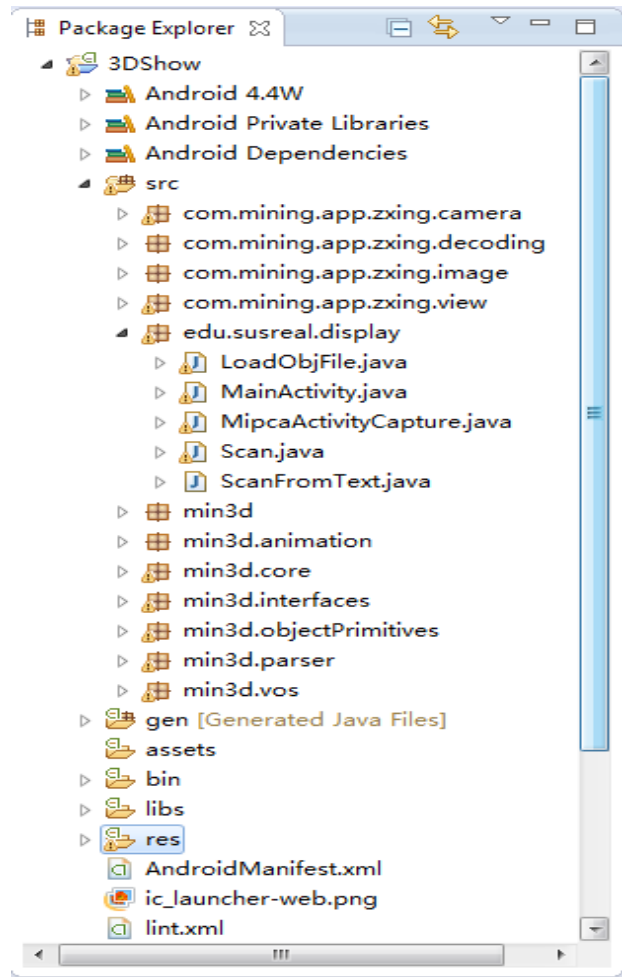

Figure. 5 Engineering Structure 


\section{The Realization of the System-3D Show}

Fig. 6. a shows the main interface. There are three methods to show the model, but we will discuss the method of scanning QR codes to loading the model from server (Fig.6.b). After scanning, the interface (Fig.6.c) includes 5 parts.

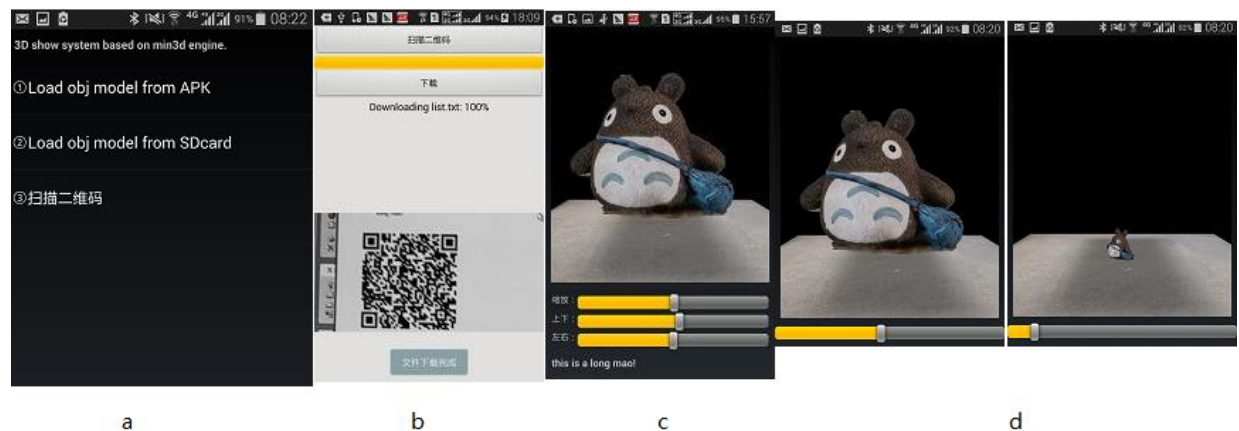

Figuer. 6 Interface. a) main interface, b) scanning QR codes and loading, c) Model showing interface, $d$ ) the comparison between origin side and zoom side

Model showing part: Most of the screen is the showing part. Its background is black, and the model shows rotating around $\mathrm{Z}$ axis.

Zoom slider: The first yellow slider in the bottom is the zoom slider. The zoom range is from 0 to10 times. The origin size is 1.0 times. Fig.6.d is the comparison between origin side and zoom side.

Up and low slider: The second yellow slider in the bottom.

Right and left slider: The third yellow slider in the bottom.

Text area

Fig.7 shows that the system displays the 3D model all-round. Besides, several different 3D models have been tested as well. The tests indicate that the software shows the plenty details and it analyzes the 3D model quickly and efficiently. All the tests go steady and the results are excellent. Fig. 8 is one of the examples.
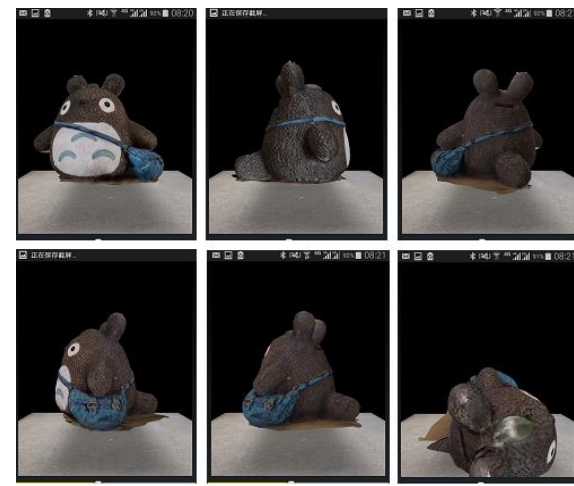

Figuer. 7 The 3D Model All-round
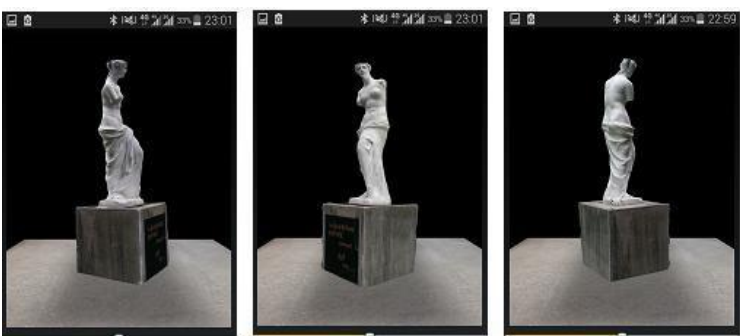

Figuer. 8 3D Model of a Large Sculpture Exhibition 


\section{Prospect and Further Work}

Although the system is designed for the demands of tourists, it still has a wide using prospect, such as showing items in the electric mall and educating etc.

Because of the limited time, this system only suits to users of Android system. The system for users of apples will be designed and developed in the future.

\section{Reference}

[1] D. G. Lowe. Distinctive image features from scale-invariant key points [J]. International Journal of Computer Vision, (2004)No.11,p.27.

[2]Jun Zhou. Multiple View Image Three Dimensional Reconstruction of Several Key Technology Research (in Chinese) （PhD, University of electronic science and technology, 2013 ),p.37.

[3]Ming Yu, Feifei Qi, Yang Yu, Gang Yan, Cuihong Xue. 3D Reconstruction based on Stereovision (in Chinese) [J]. Computer Engineering and Design, (2013) No.02, p.730-733.

[4]Shicheng Zhang. Development and Research of Application Based on Google Android (in Chinese) [J]. Computer Knowledge and Technology, (2009)No.28,p.7959-7962.

[5]YangXin,ZhaoLei. Based on a real-time multi-core architecture big picture browsing technology [J]. Journal of Image and Graphics,(2012) No.2,p.152-160.

[6] ZHANG Yanan, ZHU Changqing, DU Fuguang. Ary image resolution wavelets transform to reduce the quantitative method [J]. Journal of Geo-Information Science,2012 No.3,p.10-17.

[7] Jacke Neider, C S T ill an. Image V is ion Library Programming Guide [M]. ( CA, USA: Silicon Graphics, 1992),007-020.

[8] O. Faugeras, Q. T. Luong, T. Papadopoulo. The geometry of multiple images: the laws that govern the formation of multiple images of a scene and some of their applications [M].(Cambridge, USA: Massachusetts Institute of Technology Press, 2001),210-282

[9] S. Gauglitz, T. H llerer, M. Turk. Evaluation of interest point detectors and feature descriptors for visual tracking [J]. International journal of computer vision, (2011)No. 94,p. 335-360

[10] H. Bay, A. Ess, T. Tuytelaars, et al. Speeded-up robust features (SURF) [J]. Computer vision and image understanding, (2008) No.110(3),p.346-359 